\title{
Small Area Primary Care Physician Supply is Associated with Fewer Child Emergency Department Visits for Asthma
}

\author{
Keenan Ramsey, BSPH; Gregory D. Stevens, Ph.D., MHS \\ University of Southern California, Keck School of Medicine
}

Background: Inadequate primary care physician supply remains a major policy challenge in the U.S. and in many developed countries. Inadequate primary care supply has been previously linked with poorer health and lower life expectancy for adults. The role in child health has not been well studied despite the particularly large role that primary care plays in promoting child health, especially in managing common conditions like asthma. This study examines the relationship of physician supply (primary and specialty care) with a key measure of inadequate asthma control--asthma emergency department (ED) visits.

Methods: We used 2012 ZIP code (i.e., small area geographic postal code) level data in California to conduct cross-sectional analysis of the relationship of primary care physician supply and specialist physician supply with asthma emergency department visits in children 0-17 years of age. We included only ZIP codes with at least 5,000 residents $(n=1,184)$. Negative binomial regression was used for the analysis of non-normally distributed count data, and incident rate ratios (IRRs) are presented.

Measures: ED visit data were obtained from the California Office of Statewide Health Planning and Development. Physician data were obtained from the California Medical License Board. Primary care physicians were family medicine, general pediatrics, general internal medicine, and general practice. Specialists were all other specialties. U.S. Census Bureau covariates included major sociodemographic factors shown to drive asthma health care use in other studies. Air pollution data was obtained from the California Office of Environmental Health Hazard Assessment. See Figure 1.

Figure 1. Study Variables and Map of Analyses

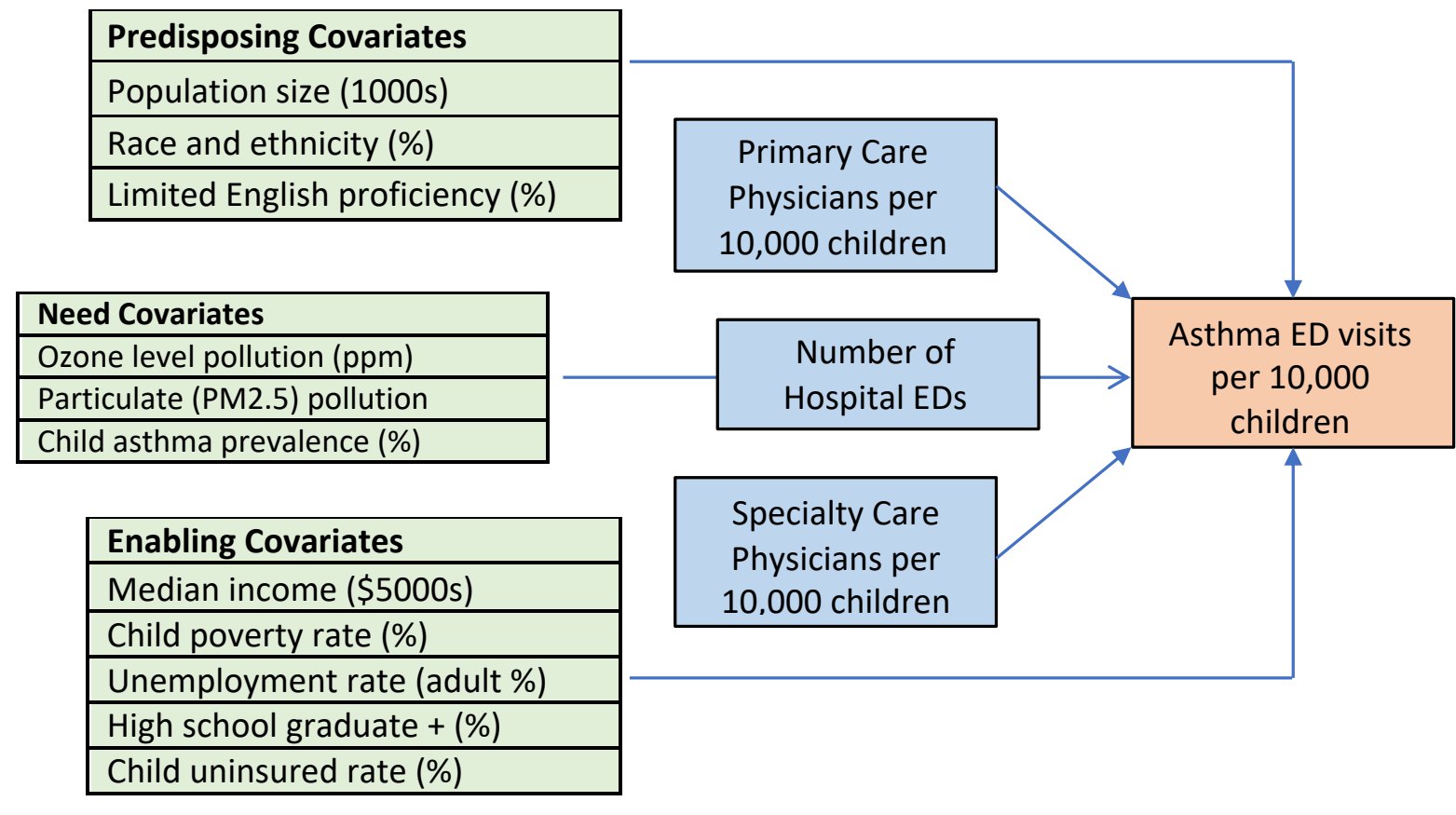

Note: Green boxes are study covariates, blue boxes are independent variables, and orange box is the dependent variable. Primary care is: family medicine, general pediatrics, general internal medicine, and general practice.
Results: Before and after controlling for the study covariates, an increase of one primary care MD per 10,000 children was associated with a $1 \%$ decrease in the asthma ED visit rate (IRR=0.99, $p<0.001)$ in a ZIP code. After adjustment (but not before), the specialist MD rate was significantly associated with a higher $E D$ visit rate $(I R R=1.01, p=0.000)$. Other predictors of ED visits included the number of hospital EDs in a ZIP code $(I R R=1.28, \quad p=0.001)$, median family income (IRR=0.94, $p=0.000)$ and child asthma prevalence rate $(I R R=1.04, p=0.001)$. Adjusted results are presented in Table 1.

Conclusions: Our study suggests that greater primary care physician supply is associated with fewer child asthma ED visits, both before and after adjusting for major predisposing, enabling, and need factors that drive health care utilization. Specialist supply was not associated with ED visits before adjustment, and significantly associated with more ED visits after controlling for the study covariates. This is notable considering the growing imbalance of primary care and specialty care in most developed and developing countries, including the U.S.

Table 1. Multivariable Negative Binomial Regression $(n=1,184)$

\begin{tabular}{|c|c|c|c|}
\hline & \multicolumn{3}{|c|}{$\begin{array}{c}\text { Asthma ED Visit Rate } \\
\text { (Adjusted) }\end{array}$} \\
\hline & IRR & $\mathrm{SE}$ & p-value \\
\hline \multicolumn{4}{|l|}{ Provider Supply } \\
\hline Primary care MDs per 10,000 & 0.99 & 0.00 & 0.000 \\
\hline Specialist MDs per 10,000 & 1.01 & 0.01 & 0.000 \\
\hline Number of hospitals with EDs & 1.28 & 0.09 & 0.001 \\
\hline \multicolumn{4}{|l|}{ Predisposing Covariates } \\
\hline Population size (1000s) & 1.02 & 0.01 & 0.002 \\
\hline \multicolumn{4}{|l|}{ Race and ethnicity (\%) } \\
\hline -Asian & 0.99 & 0.01 & 0.182 \\
\hline -Black & 1.00 & 0.01 & 0.990 \\
\hline -Hispanic/Latino & 1.00 & 0.01 & 0.717 \\
\hline -White (non-Hispanic) & 0.98 & 0.01 & 0.008 \\
\hline Limited English proficiency (\%) & 1.00 & 0.01 & 0.582 \\
\hline \multicolumn{4}{|l|}{ Enabling Covariates } \\
\hline Median income (\$5000s) & 0.94 & 0.01 & 0.000 \\
\hline Child poverty rate (\%) & 1.01 & 0.01 & 0.105 \\
\hline Unemployment rate (\%) & 0.98 & 0.01 & 0.195 \\
\hline High school graduate + (\%) & 1.02 & 0.01 & 0.009 \\
\hline Child uninsured rate (\%) & 0.99 & 0.01 & 0.158 \\
\hline \multicolumn{4}{|l|}{ Need Covariates } \\
\hline Ozone level pollution (ppm) & 1.09 & 0.19 & 0.596 \\
\hline Particulate (PM2.5) pollution & 1.01 & 0.01 & 0.168 \\
\hline Child asthma prevalence (\%) & 1.04 & 0.01 & 0.001 \\
\hline
\end{tabular}

